\title{
Prevalência de excesso de peso e fatores associados em uma região metro- politana do Nordeste brasileiro
}

\author{
Prevalence of overweight and associated factors in a metropolitan area in the Northeast \\ of Brazil
}

Ariel Figueiredo Oliveira ${ }^{1}$, Augusto César Santiago Araújo Júnior ${ }^{1}$, Josilda Ferreira Cruz ${ }^{1}$, Mário Augusto Ferreira Cruz, Demetrius Silva de Santana², Karla Freire Rezende², Cristiane Costa da Cunha Oliveira ${ }^{1}$, Sônia Oliveira Lima ${ }^{1}$

\section{RESUMO}

Objetivo: Identificar o perfil clínico e sociodemográfico de adultos com excesso de peso na cidade de Aracaju, Sergipe. Estudo transversal, descritivo tipo survey, com abordagem analítica quantitativa. Método: Amostra com 690 indivíduos que procuraram, de forma aleatória, 4 centros de referência para a realização de exame de ultrassonografia abdominal. A aferição de medidas antropométricas ocorreu entre julho de 2013 e julho de 2014. Foram analisadas as variáveis: idade, sexo, renda, escolaridade, comorbidades, circunferência da cintura e índice de massa corporal (IMC). O programa estatístico utilizado foi o Statistical Package for the Social Sciences (SPSS ${ }^{\circledR}$ ) versão 22.0 e o nível de significância considerado foi $\mathrm{p}<0,05$. Resultados: 494 mulheres $(71,6 \%)$ e 196 homens $(28,4 \%)$ foram avaliados. A média de idade dos pacientes foi de 39,4 anos e, naqueles com obesidade, foi de 42,3 anos. Em relação ao IMC, 52,9\% dos indivíduos eram eutróficos, $31,2 \%$ tinham sobrepeso e o restante era classificado como portador de obesidade grau I (10,4\%), grau II $(4,5 \%)$ ou grau III (1,0\%). O IMC foi significativamente maior no grupo dos hipertensos $(\mathrm{p}=0,001)$, no grupo com menor escolaridade $(\mathrm{p}=0,004)$, bem como no grupo com maior renda $(\mathrm{p}=0,003)$. A circunferência da cintura apresentou média de 104,9 cm em obesos. Conclusão: Observaram-se altas prevalências de excesso de peso nos adultos pesquisados, dado consoante à tendência epidemiológica nacional, o que permite classificar a situação como um problema de saúde pública no município avaliado.

Palavras-chave: peso corporal; sobrepeso; obesidade; índice de massa corpórea; epidemiologia; estudos transversais.

\begin{abstract}
Objective: This article aimed to identify the clinical and socio-demographic profile of overweight adults evaluated through survey and anthropometric measurements in the city of Aracaju, Sergipe, Brazil. Methods: Descriptive transversal outline survey-type study in a quantitative analytical approach. The sample was composed of 690 individuals who randomly sought four reference centers in the municipality of Aracaju for abdominal ultrasonography. Anthropometric measurements were taken between July 2013 and July 2014. The following variables were analyzed: age, gender, income, schooling, comorbidities, waist girth and body mass index (BMI). The statistical program Statistical Package for the Social Sciences (SPSS) 22.0 was chosen, and significance level was p<0.05. Results: 494 women (71.6\%) and 196 men (28.4\%) were analyzed. The average age was 39.4 years, and 42.3 years for obese patients. Regarding BMI, $52.9 \%$ of patients presented normal BMI; $31.2 \%$ were overweight; $10.4 \%$ presented level 1 obesity; $4.5 \%$, level 2; and $1.0 \%$, level 3 . The BMI was significantly higher among the hypertensive ones $(\mathrm{p}=0.001)$, with poor schooling $(\mathrm{p}=0.004)$, and those with higher income ( $\mathrm{p}=0.003$ ). Average waist circumference was $104.94 \mathrm{~cm}$ among the obese portion. Conclusion: The observed high prevalence of overweight in the studied adults is in accordance with the national epidemiologic tendency, which allows classifying the situation as a public health problem in the evaluated municipality.
\end{abstract}

Keywords: body weight; overweight; obesity; body mass index; epidemiology; cross-sectional studies.

${ }^{1}$ Universidade Tiradentes, Faculdade de Medicina - Aracaju (SE), Brasil.

${ }^{2}$ Universidade Federal de Sergipe, Faculdade de Medicina - Aracaju (SE), Brasil.

Autor correspondente: Josilda Ferreira Cruz - Avenida Jornalista Santos Santana, 490 - Jardins - CEP: 49025-100 - Aracaju

(SE), Brasil - E-mail:arielfig1995o@ gmail.com

Recebido em 06/06/2016. Aceito para publicação em 08/03/2017. 


\section{INTRODUÇÃO}

Nos dias atuais, a obesidade, em razão de sua magnitude, é considerada a mais importante desordem nutricional nos países desenvolvidos e em desenvolvimento, devido ao aumento da sua incidência, o que a fez atingir proporção epidêmica mundial, caracterizando, portanto, uma relevante problemática para a saúde pública. ${ }^{1,2}$

A obesidade pode ser definida como um agravo de etiologia multifatorial, cuja principal causa se refere a um balanço energético positivo, ou seja, o indivíduo consome mais energia do que gasta, o que gera excesso de gordura corporal sob a forma de tecido adiposo. Diversos trabalhos buscam explicar a complexa etiologia da obesidade e descrevem como suas principais causas fatores históricos, ecológicos, políticos, sociais, culturais, genéticos, metabólicos e psíquicos. ${ }^{1,3}$

A Organização Mundial de Saúde (OMS) recomenda a utilização do índice de massa corporal (IMC) para o diagnóstico do estado nutricional de grupos populacionais para determinar o sobrepeso e a obesidade. ${ }^{4}$ Por se correlacionar bem à gordura corporal, ser de fácil mensuração, não ser uma medida invasiva e ser de baixo custo, é o indicador mais amplamente usado. ${ }^{5}$ Em adultos, um IMC igual ou superior a $25 \mathrm{~kg} / \mathrm{m}^{2}$ é caracterizado como sobrepeso, e igual ou superior a $30 \mathrm{~kg} / \mathrm{m}^{2}$ configura obesidade. O IMC, além de classificar o indivíduo com relação ao peso, também é um indicador de riscos para a saúde e tem relação com várias complicações metabólicas. ${ }^{6-8}$

Nos dias atuais, o panorama mundial e brasileiro das chamadas doenças crônicas não transmissíveis (DCNT) revela-se como novo desafio para a saúde pública. ${ }^{5}$ Nesse grupo de doenças, a obesidade ocupa lugar de destaque, visto que, além de ser uma doença em si, é fator de risco para as referidas DCNT, principalmente doenças cardíacas, acidente vascular encefálico, câncer, doenças respiratórias crônicas e diabetes mellitus (DM). Em consequência, a OMS caracteriza tal agravo como epidemia global que atinge as mais variadas faixas etárias em países desenvolvidos ou em desenvolvimento. ${ }^{8-11}$

O entendimento sobre como se comportam o excesso de peso e a obesidade no país indica haver uma necessidade de concreta definição das prioridades e das estratégias de ações públicas direcionadas à prevenção e ao controle desses agravos, com especial destaque para as medidas de educação em saúde e nutrição, em âmbitos regional e nacional, assim como em todos os segmentos da sociedade. Dessa forma, o presente estudo pretendeu avaliar na cidade de Aracaju, Sergipe, o perfil clínico e sociodemográfico de adultos com excesso de peso, avaliados por meio de questionário e medidas antropométricas, e, a partir dos resultados encontrados, sugerir a adoção de medidas de promoção da saúde e prevenção de agravos.

\section{MÉTODOS}

Trata-se de um estudo de delineamento transversal de base populacional, não experimental, descritivo tipo survey, com abordagem analítica quantitativa. A população foi escolhida por intermédio de um processo amostral aleatório.

Os dados foram coletados em quatro clínicas de especialidades no município de Aracaju, Sergipe, no período de julho de 2013 a julho de 2014, após aprovação do Comitê de Ética em Pesquisa da Universidade Tiradentes, situada em Aracaju, Sergipe, conforme regulamentação editada pela Resolução no 196/96, da Comissão Nacional de Ética em Pesquisa (CONEPE), e permissão da utilização do espaço físico dos serviços privados utilizados.

Para o cálculo do tamanho da amostra, considerou-se uma prevalência de $21 \%$ de obesidade, de acordo com o estudo de Gigante et al., ${ }^{12}$ considerando o erro de 5\%. Com base na fórmula de Pocock ${ }^{13}$ :

$\mathrm{E}^{2}=\alpha^{2} \cdot \mathrm{p} \cdot \mathrm{q} / \mathrm{n}$

Em que:

$\mathrm{E}=$ erro amostral;

$\mathrm{p}=$ prevalência;

$\mathrm{q}=$ complemento da prevalência; e $\alpha=1,96$,

A amostra calculada foi de 255 pacientes, com perda de $10 \%$, totalizando uma amostra final de 280 .

Foram utilizados como critérios de inclusão na pesquisa: ter procurado, de forma aleatória, um dos quatro serviços de referência para realização de ultrassonografia abdominal; adultos, de ambos os sexos, de 18 a 60 anos de idade; e assinar a permissão para o preenchimento do questionário. Utilizou-se como critério de exclusão: não ser capaz de responder ao questionário por deficiência mental.

Todos os participantes, após a assinatura do Termo de Consentimento Livre e Esclarecido (TCLE), responderam a um questionário com informações sociodemográficas e realizaram aferição de medidas antropométricas. O referido questionário continha informações sobre as seguintes variáveis: nome, idade, data de nascimento, sexo, naturalidade, escolaridade, renda, comorbidades associadas como DM tipo 2 e hipertensão arterial sistêmica (HAS).

A avaliação antropométrica consistiu na aferição de peso, altura e circunferência da cintura. Para o peso corpóreo, foi utilizada uma balança portátil marca TECHLINE com o paciente em pé, descalço, no centro da balança, com o peso distribuído em ambos os pés. A aferição da altura foi feita por meio de um estadiômetro portátil da marca Filizola, com a leitura realizada no mm mais próximo, com o paciente descalço, com os braços estendidos verticalmente ao longo do corpo, os ombros relaxados, os calcanhares juntos e a cabeça posicionada.

Os dados de peso e altura foram utilizados para o cálculo do IMC, medido pelo índice de Quetelet, pela razão entre o peso corpóreo $(\mathrm{em} \mathrm{kg})$ e a altura $\left(\mathrm{em} \mathrm{m}^{2}\right)$, e classificado segundo critério da OMS em: até 24,9, normal; de 25 a 29,9, sobrepeso; de 30 a 34,9, obesidade grau I ou leve; de 35 a 39,9 , obesidade grau II ou moderada; e $\geq 40$, obesidade grau III ou grave. ${ }^{6,7}$ A circunferência da cintura foi medida com o paciente em pé, utilizando uma fita métrica inelástica no nível do ponto médio entre a última costela e a crista ilíaca. ${ }^{14}$

Cada variável estudada - sexo, idade, escolaridade, renda, circunferência da cintura e comorbidades - foi comparada com os valores do IMC. Os testes $t$ de Student e ANOVA com pós-teste de Tukey foram executados para as 
comparações entre os grupos formados. Os valores estão expressos em média e desvio padrão (DP) da média. A significância estatística foi estipulada em 5\% (p<0,05). Para todas as análises foi utilizado o programa Statistical Package for the Social Sciences $\left(\right.$ SPSS $^{\circledR}$ ) versão 22.0.

\section{RESULTADOS}

Foi analisado um total de 690 indivíduos. A média de idade observada no grupo como um todo foi de 39,5 anos $(\mathrm{DP}=11,3)$, sendo 43,1 anos ( $\mathrm{DP}=10,2)$ naqueles com sobrepeso e de 42,4 anos (DP=9,8) nos obesos. Os dados dos indivíduos estudados, de acordo com a distribuição por sexo, IMC, renda e escolaridade, estão apresentados na Tabela 1.

A circunferência da cintura apresentou média de $85,52 \mathrm{~cm}(\mathrm{DP}=13,26)$ no indivíduos como um todo; e em obesos a média foi de 104,94 cm (DP=10,57).

Os valores de IMC comparados entre sexos, rendas, níveis de escolaridade e comorbidades são apresentados na Tabela 2.

O IMC foi significativamente maior no grupo dos hiperten$\operatorname{sos}(\mathrm{p}=0,001)$; não houve associação estatisticamente significativa entre IMC e DM ( $\mathrm{p}=0,14)$. Para escolaridade e IMC houve associação estatisticamente significativa $(\mathrm{p}=0,004)$, com IMC mais elevado no grupo com menor escolaridade (Tabela 2 e Figura 1).

Tabela 1. Amostra total dos indivíduos pesquisados, categorizados segundo sexo, índice de massa corporal, renda e escolaridade.

\begin{tabular}{lcc} 
Variáveis & n & \% \\
\hline Sexo & & \\
F & 494 & 71,6 \\
M & 196 & 28,4 \\
\hline IMC & & \\
$\quad<25$ & 365 & 52,9 \\
$\geq 25$ e $<30$ & 215 & 31,2 \\
$\geq 30$ e $<35$ & 72 & 10,4 \\
$\geq 35$ e $<40$ & 31 & 4,5 \\
$\geq 40$ & 7 & 1,0 \\
\hline Renda (salários mínimos) & & \\
Abaixo de 1,5 & 437 & 63,3 \\
De 1,5 a 4,5 & 206 & 29,9 \\
Acima de 4,5 & 47 & 6,8 \\
\hline Escolaridade & & \\
Até ensino fundamental & 246 & 35,7 \\
Até ensino médio & 254 & 36,8 \\
Ensino superior & 189 & 27,4 \\
Não soube informar & 1 & 0,1 \\
\hline
\end{tabular}

Tabela 2. Associação das médias do índice de massa corporal com variáveis como sexo, comorbidades (hipertensão arterial sistêmica e diabetes mellitus), renda e escolaridade.

\begin{tabular}{lcccc} 
& \multicolumn{3}{c}{ IMC } & Valor \\
\cline { 2 - 4 } & Média & DP & n & p \\
Sexo & & & & \\
F & 24,95 & 5,5 & 494 & 0,066 \\
M & 25,77 & 4,8 & 196 & \\
HAS $^{a}$ & & & & \\
Não & 24,65 & 5,0 & 589 & \\
Sim & 29,1 & 5,7 & 89 & $\mathbf{0 , 0 0 1}$ \\
DM $^{a}$ & & & & \\
Não & 25,2 & 5,17 & 657 & \\
Sim & 26,9 & 7,92 & 21 & 0,141 \\
Renda (salários mínimos) & & & & \\
Abaixo de 1,5 & 24,67 & 5,49 & 437 & \\
De 1,5 a 4,5 & 25,97 & 4,83 & 206 & $\mathbf{0 , 0 0 3}$ \\
Acima de 4,5 & 26,45 & 4,78 & 47 & \\
\hline Escolaridade & & & & \\
Até ensino fundamental & 26,06 & 5,33 & 246 & \\
Até ensino médio & 24,66 & 5,21 & 254 & $\mathbf{0 , 0 0 4}$ \\
Ensino superior & 24,7 & 5,23 & 189 & \\
\hline
\end{tabular}

IMC: índice de massa corporal; DP: desvio padrão; HAS: hipertensão arterial sistêmica; DM: diabetes mellitus tipo 2; p: nível de significância; ${ }^{a} 12$ pacientes não souberam informar; ${ }^{b} 1$ paciente não soube informar sua escolaridade.

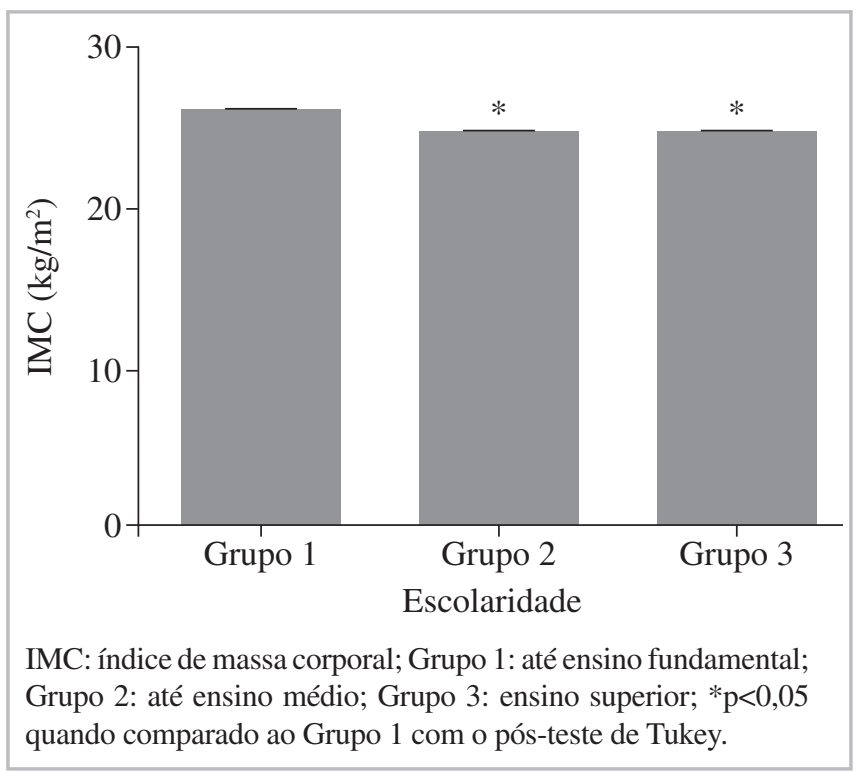

Figura 1. Associação entre escolaridade e índice de massa corporal. 
Houve associação estatisticamente significativa entre renda e IMC ( $p=0,003)$, tendo sido observado maior IMC nos grupos de maior renda (Tabela 2 e Figura 2).

\section{DISCUSSÃO}

Wanderley e Ferreira ${ }^{1}$ apontam que a obesidade é, atualmente, considerada a mais importante desordem nutricional nos países desenvolvidos e em desenvolvimento. Pêgo-Fernandes et al. ${ }^{15}$ relataram que aproximadamente 1,6 bilhão de indivíduos com idade superior a 15 anos estavam em situação de sobrepeso e 400 milhões eram obesos. De acordo com os dados da última Pesquisa de Orçamentos Familiares $(\mathrm{POF})^{16}$ feita no Brasil, cerca de $15 \%$ dos adultos apresentam obesidade e cerca de metade da população maior de 20 anos apresenta excesso de peso. Em concordância com os dados citados, a última pesquisa feita por meio da Vigilância de Fatores de Risco e Proteção para Doenças Crônicas por Inquérito Telefônico (VIGITEL) ${ }^{17}$ indicou crescimento do número de pessoas com excesso de peso no país: $52,5 \%$ dos brasileiros estão acima do peso (o índice era de 43\% em 2006) e $17,9 \%$ da população está obesa. O presente estudo não difere da tendência mundial e nacional, já que foram evidenciadas altas prevalências de excesso de peso em adultos de ambos os sexos $(47,11 \%)$, o que permite classificar a situação como um problema de saúde pública no município avaliado (Aracaju).

A POF de 2008-2009 ${ }^{16}$ demonstra dados do Brasil para excesso de peso segundo o sexo, com prevalência de 50,1\% para os homens e $48 \%$ para as mulheres. Resultado semelhante ao citado foi encontrado pelo VIGITEL Brasil, ${ }^{17}$ que apresentou prevalência de excesso de peso de $49,1 \%$ em mulheres e 56,5\% em homens. Os dados encontrados na presente pesquisa, ao relacionar as prevalências de excesso de peso segundo o sexo no município de Aracaju, indicaram maior

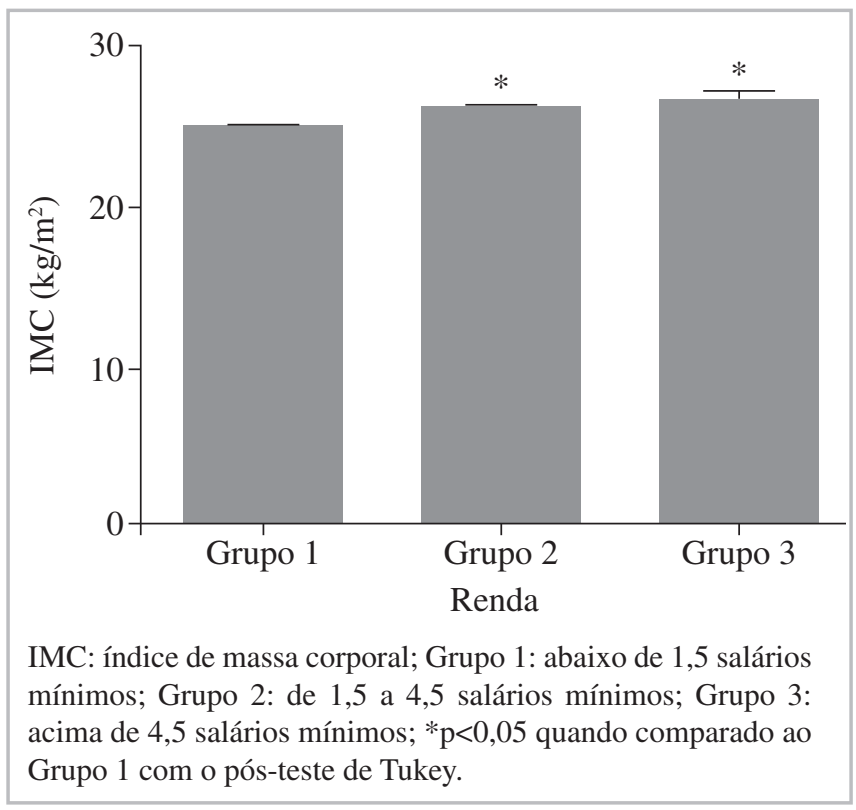

Figura 2. Associação entre índice de massa corporal e renda. prevalência em homens, com uma média de IMC de 25,77, e menor prevalência em mulheres, com uma média de IMC de 24,95 , o que identifica uma possível tendência epidemiológica conforme apontam as importantes pesquisas nacionais já citadas, além de inferir, haja vista o maior número de mulheres como sexo pesquisado, maior preocupação desse grupo com a saúde e, consequentemente, menor prevalência de excesso de peso. ${ }^{16,17}$

Holanda et al., ${ }^{18} \mathrm{em}$ suas pesquisas, relataram aumento linear do excesso de peso à medida que aumenta a idade. Segundo o estudo, a partir dos 40 anos a prevalência de excesso de peso esteve presente em maior frequência. Guimarães et al. ${ }^{19} \mathrm{e}$ Machado et al..$^{20}$ também encontraram dados semelhantes no que se refere ao aumento da idade ser diretamente proporcional a maiores chances de haver excesso de peso. O VIGITEL ${ }^{17}$ também aponta que a prevalência de excesso de peso acompanha o aumento da idade. A pesquisa relata que na faixa etária de 18 a 24 anos o percentual foi de $31,5 \%$ e na faixa etária de 55 a 64 anos o percentual foi de $61,8 \%$.

No que se refere à relação entre excesso de peso e escolaridade, Backes et al..$^{21}$ identificaram em suas pesquisas na zona urbana do município de São Leopoldo, Rio Grande do Sul, que o grupo de maior percentual para excesso de peso pertencia àqueles que foram classificados como indivíduos com baixa escolaridade $(61,7 \%)$. Os recentes dados do VIGITEL, ${ }^{17}$ divulgados em 2015, corroboram a pesquisa anteriormente citada ao afirmar que quanto menor a escolaridade, maior a prevalência do excesso de peso. O estudo recente de Rodrigues e Silveira, ${ }^{22}$ avaliando obesos graves, encontrou correlação negativa significante de escolaridade com peso $(\mathrm{r}=-0,234)$ e IMC $(\mathrm{r}=-0,364)$. O presente estudo aponta a mesma tendência das importantes pesquisas citadas, já que menos anos de estudo estiveram relacionados à presença de excesso de peso.

Silva et al., ${ }^{23} \mathrm{em}$ seu estudo sobre a prevalência e os fatores associados ao excesso de peso em adultos do Brasil, identificaram significativa associação entre renda e adiposidade. Os autores classificaram a renda em alta, média ou baixa (maior ou igual a 4 salários mínimos, igual a 4 salários mínimos e entre 2 e 4 salários mínimos, respectivamente) e os indivíduos pesquisados com excesso de peso/obesidade possuíam, em sua maioria, renda qualificada como alta $(29,4 \%$ estavam com sobrepeso e 10,8\% eram obesos). Lino et al., ${ }^{24}$ de maneira semelhante, relataram em seus estudos que, dos indivíduos com excesso de peso, a maioria possuía uma renda de mais de três salários mínimos. Rodrigues e Silveira ${ }^{22}$ encontraram que em obesos graves a maior renda per capita se associou ao maior consumo de vegetais folhosos e de doces. A presente pesquisa apontou a mesma tendência da literatura, já que maiores salários se relacionaram com os maiores valores de IMC.

Já está bem estabelecida na literatura a íntima relação entre valores aumentados de IMC e a concomitância com a HAS. Segundo Amer et al. ${ }^{25}$, em âmbito mundial a prevalência da hipertensão arterial está muito mais aumentada em indivíduos com peso acima dos valores normais do que na- 
queles que estão com o peso na faixa de normalidade. Girotto et al..$^{26}$ identificaram em suas pesquisas alta prevalência e correlação entre obesidade e hipertensão. Da mesma forma, Cavagioni e Pierin ${ }^{27}$ identificaram associação significativa entre hipertensão e maiores níveis de IMC, dados que corroboram os resultados encontrados no presente estudo.

Nos indivíduos com excesso de peso, a ação da insulina na gênese do DM já está bem elucidada, conforme explicitam Swarowsky et al. ${ }^{2}$ ao afirmarem que o aumento dos níveis glicêmicos e a hiperinsulinemia gerados a partir da obesidade baseiam-se no fato de que o tecido adiposo eleva a demanda de insulina, gera resistência a ela mesma e tem como consequência o diabetes. A inter-relação entre obesidade e DM é tão grande, aponta Luft, ${ }^{28}$ que o termo "diabesidade" tem sido empregado para enfatizar a importância da obesidade como fator etiopatogênico do DM. Torres et al. ${ }^{29}$ encontraram em seus estudos associação significativa entre excesso de peso e DM. Em uma de suas pesquisas, apontaram que homens e mulheres portadores de diabetes possuíam média de IMC de 28,91 e 30,54 , respectivamente. O presente estudo também evidenciou aumento do IMC nos portadores de DM, muito embora não tenha comprovado associação estatisticamente significativa $(\mathrm{p}=0,14)$, já que o número de pacientes com DM foi baixo $(n=21)$.

A prevalência de excesso de peso e obesidade da amostra populacional avaliada na cidade de Aracaju foi alta, sendo maior no sexo masculino, em classes sociais mais altas e de menor escolaridade. A associação com HAS foi evidenciada. Embora existam diversos métodos para avaliação da obesidade, a presente pesquisa apenas avaliou como indicador antropométrico o IMC. Apesar do emprego desse único dado, o instrumento mostrou-se de fácil utilização, de baixo custo e com resultados consistentes que corroboraram pesquisas nacionais e internacionais acerca do excesso de peso em populações. Dessa forma, possibilitou a identificação de grupos mais vulneráveis e, assim, poderá permitir a adoção de medidas de promoção da saúde e prevenção de agravos em determinados grupos, haja vista o impacto que o excesso de peso pode gerar na saúde pública.

Portanto, sugere-se a realização de outros estudos sobre excesso de peso e obesidade, a fim de caracterizar novos fatores de risco biopsicossociais e, assim, ser possível a adoção de medidas preventivas de saúde pública, diminuindo a prevalência do excesso de peso e da obesidade na população.

\section{REFERÊNCIAS}

1. Wanderley EN, Ferreira VA. Obesidade: uma perspectiva plural. Ciênc Saúde Coletiva. 2010;15(1):185-94. DOI: 10.1590/S1413-81232010000100024

2. Swarowsky I, Reuter EM, Ferreira C, Priebe P, Paiva DN, Pohl $\mathrm{HH}$. Obesidade e fatores associados em adultos. Cinergis. 2012;13(1):64-71. DOI: 10.17058/cinergis.v13i1.2845

3. Sarturi JB, Neves J, Peres KG. Obesidade em adultos: estudo de base populacional num município de pequeno porte no sul do Brasil em 2005. Ciênc Saúde
Coletiva. 2010;15(1):105-13. DOI: 10.1590/S141381232010000100016

4. Barbosa Filho VC, Quadros TMB, Souza EA, Gordia AP, Campos W. A utilização do critério da Organização Mundial de Saúde para classificação do estado nutricional em crianças. Motriz Rev Educ Fís. 2010;16(4):811-9. DOI: 10.5016/1980-6574.2010v16n4p811

5. Brasil. Ministério da Saúde. Secretaria de Atenção à Saúde. Departamento de Atenção Básica. Estratégias para o cuidado da pessoa com doença crônica: obesidade. Brasília: Ministério da Saúde; 2014.

6. Pombo F, Monteiro C, Pecorelli C, Antunes JL. Caracterização dos alunos de Ciências e Tecnologias da Saúde em relação à actividade física, índice de massa corporal e hábitos alimentares. O caso da ERISA. Rev Lusófona Ciênc Tecnol Saúde. 2010;7(1):37-46.

7. Rezende FAC, Rosado LEFPL, Franceschinni SCC, Rosado GP, Ribeiro RCL. Aplicabilidade do índice de massa corporal na avaliação da gordura corporal. Rev Bras Med Esporte. 2010;16(2):90-4. DOI: 10.1590/ S1517-86922010000200002

8. Oliveira ML. Estimativa dos custos da obesidade para o Sistema Único de Saúde do Brasil [tese]. Brasília: Universidade de Brasília, Departamento de Nutrição Humana; 2013.

9. Ferreira SRG. Alimentação, nutrição e saúde: avanços e conflitos da modernidade. Ciênc Cult. 2010;62(4):31-4.

10. Freitas LKP, Cunha Júnior AT, Knackfuss MI, Medeiros HJ. Obesidade em adolescentes e as políticas públicas de nutrição. Ciênc Saúde Coletiva. 2014;19(6):175562. DOI: $10.1590 / 1413-81232014196.12382013$

11. Schmidt MI, Duncan BB, Silva GA, Menezes AM, Monteiro CA, Barreto SM, et al. Chronic noncommunicable diseases in Brazil: burden and current challenges. Lancet. 2011;377(9781):1949-61. DOI: 10.1016/S0140-6736(11)60135-9

12. Gigante DP, Barros FC, Post CLA, Olinto MTA. Prevalência de obesidade em adultos e seus fatores de risco. Rev Saúde Pública. 1997;31(3):236-46. DOI: 10.1590/S0034-89101997000300004

13. Pocock SJ. Clinical trials: a practical approach. Chinchester: John Wiley \& Sons; 1983. p.123-41.

14. Lohman TG, Roche AF, Martorell R. Anthropometric standardization reference manual. Champaign: Human Kinetics; 1988. v.1, p.124.

15. Pêgo-Fernandes PM, Bibas BJ, Deboni M. Obesity: the greatest epidemic of the 21st century? São Paulo Med J. 2011;129(5):283-4. DOI: 10.1590/S151631802011000500001

16. Instituto Brasileiro de Geografia e Estatística. Pesquisa de Orçamentos Familiares 2008-2009: antropometria e estado nutricional de crianças, adolescentes e adultos no Brasil. Rio de Janeiro: IBGE; 2010.

17. Brasil. Ministério da Saúde. Vigilância de fatores de risco e proteção para doenças crônicas por inquérito telefônico (VIGITEL). Brasília: Ministério da Saúde; 2014. 
18. Holanda LGM, Martins MCC, Souza Filho MD, Carvalho CMRG, Assis RC, Leal LMM, et al. Excesso de peso e adiposidade central em adultos de Teresina-PI. Rev Assoc Méd Bras. 2011;57(1):50-5. DOI: 10.1590/ S0104-42302011000100016

19. Guimarães NG, Dutra ES, Ito MK, Carvalho KMB. Adesão a um programa de aconselhamento nutricional para adultos com excesso de peso e comorbidades. Rev Nutr. 2010;23(3):323-33. DOI: 10.1590/S141552732010000300001

20. Machado IE, Pereira SCL, Dias-Júnior CS, Abreu MNS, Borges AM, Filgueiras JH. Fatores associados ao excesso de peso em adultos usuários de restaurantes populares em Belo Horizonte, Brasil. Ciênc Saúde Coletiva. 2014;19(5):1367-77. DOI: 10.1590/141381232014195.21772013

21. Backes V, Olinto MTA, Henn RL, Cremonese C, Pattussi MP. Associação entre aspectos psicossociais e excesso de peso referido em adultos de um município de médio porte do Sul do Brasil. Cad Saúde Pública. 2011;27(3):573-80. DOI: 10.1590/S0102$311 X 2011000300017$

22. Rodrigues APS, Silveira EA. Correlação e associação de renda e escolaridade com condições de saúde e nutrição em obesos graves. Ciênc Saúde Coletiva. 2015;20(1):165-74. DOI: 10.1590/1413-81232014201.18982013

23. Silva VS, Petroski ED, Souza I, Silva DAS. Prevalência e fatores associados ao excesso de peso em adultos do Brasil: um estudo de base populacional em todo território nacional. Rev Bras Ciênc Esporte. 2012;34(3):713-26. DOI: 10.1590/S0101-32892012000300013

24. Lino MZR, Muniz PT, Siqueira KS. Prevalência e fatores associados ao excesso de peso em adultos: inquérito populacional em Rio Branco, Acre, Brasil, 2007-2008. Cad Saúde Pública. 2011;27(4):797-810. DOI: 10.1590/S0102-311X2011000400019

25. Amer NM, Marcon SS, Santana RG. Índice de massa corporal e hipertensão arterial em indivíduos adultos no Centro-Oeste do Brasil.ArqBras Cardiol.2011;96(1):4753. DOI: 10.1590/S0066-782X2010005000154

26. Girotto E, Andrade SM, Cabrera MAS. Prevalência de obesidade abdominal em hipertensos cadastrados em uma Unidade de Saúde da Família. Arq Bras Cardiol. 2010;94(6):754-62. DOI: 10.1590/S0066782X2010005000049

27. Cavagioni LC, Pierin AMG. Hipertensão arterial e obesidade em motoristas profissionais de transporte de cargas. Acta Paul Enferm. 2010;23(4):455-60. DOI: 10.1590/S0103-21002010000400002

28. Luft VC. Obesidade e diabetes: contribuição de processos inflamatórios e adipocitocinas, e a potencial importância de fatores nutricionais [tese]. Porto Alegre: Universidade Federal do Rio Grande do Sul, Departamento de Epidemiologia; 2010.

29. Torres HC, Pace AE, Stradioto MA. Análise sociodemográfica e clínica de indivíduos com diabetes tipo 2 e sua relação com o autocuidado. Cogitare Enferm. 2010;15(1):48-54. DOI: 10.5380/ce.v15i1.17144 